\title{
Rasmussen's encephalitis
}

\author{
Krishnan Vijayan, Madeswaran K and Ramya R \\ Royal care super speciality hospital, Coimbatore, India
}

\begin{abstract}
Adult Rasmussen encephalitis is a rare unihemispheric inflammatory disease of the brain that leads to intractable seizures, cognitive decline and progressive neurological deficits associated with the affected hemisphere. It forms only $10 \%$ of the total reported cases of rasmussen encephalitis. The autoimmune nature of the disease has led to trail of various immuno modulatory treatments for the same. Recognition of in this entity by itself is difficult and often requires brain biopsy for definitive diagnosis and treatment. Here with we report a case of biopsy proved adult rasmussen encephalitis who was treated with IV rituximab followed by oral mycophenolate and has remained seizure free in remission for the past six years.
\end{abstract}

\section{Introduction}

Rasmussen's encephalitis (RE) is rare unihemispheric inflammatory disease of the brain that leads to intractable seizures, cognitive decline and progressive neurological deficits associated with the affected hemisphere [1]. The age at onset is in childhood, between 6 and 8 years (range: 1-13 years) and affects children who were previously healthy [2]. Sometimes, an infectious or inflammatory disease has been noted during the 6 months before the first Seizure.

\section{Late onset of Rasmussen's encephalitis}

In around $10 \%$ of cases of Rasmussen's encephalitis, the disease starts after the age of 12-13 years, with onset occurring as late as 54-years. The evolution is more variable with a more insidious onset and cerebral hemi atrophy at a later stage [3].

\section{Clinical staging by-Oguni et al [4].}

Stage 1-Correlates to the development of seizures until the hemiparesis is established ( 3 months -10 years). During this stage, the seizures frequency and intensity progressively increase. seizures may transiently improve during this stage, but they tend to reoccur and become soon pharmacoresistant.

Stage 2- Characterised by a fixed hemiparesis and additional neurological deficits including intellectual decline (2 months - 10 years). Moreover, there is an increase in seizure frequency and intensity; seizures may even be continuously present. The epileptic activity appears to propagate to other sites within the same hemisphere leading to new symptoms and polymorphous seizures (motor, somatosensory, visual symptoms).

Stage 3-Marked by the stabilisation of the disease and a decrease of seizure activity. The neurological deficit is established, such as spastic hemiparesis, visual field and sensory deficits. There may be additional intellectual disability which can range from mild to severe grades.

\section{Pathogenesis}

Cytotoxic $\mathbf{t}$ cells-inverse correlation between the intensity of the inflammatory markers ( $\mathrm{T}$ lymphocytes and microglial nodules) and disease duration has been observed. Granzyme B (GrB) mediated cytotoxic T lymphocyte (CTL) attack neurons. HUMORAL- rabbits immunized with the GluR3 fusion protein develop seizures.

Immunogenic section of the GluR3 protein could be exposed to the immune system only after cleavage of GluR3 by GrB. A necessary prerequisite for this is that an internal $\mathrm{N}$ linked glycosylation sequence within the GluR3-GrBrecognition sequence (ISND ${ }^{\star}$ S) is not glycosylated.

\section{Histopathology}

Cortical inflammation with perivascular lymphocytic cuffing gliosis with microglial activation and microglial nodules, as well as astrocytes and neurons loss have been observed. Lesions tend to become confluent, in that they do not remain multifocal. The intensity of histopathological lesions becomes less with the duration of the disease, resulting in some sort of "burnout" Picture.

\section{Mri staging [5]}

1-shows no abnormality, 2-shows oedema and increased signals, 3-reveals increased signals with normal hemispheric volume,4-shows increased signals with hemispheric atrophy, 5- There is a disappearance of the increased signal, leaving a markedly atrophied cerebral hemisphere.

EEG [6]: The following unihemispheric findings strongly suggest RE: Impairment of background activity and sleep spindles; focal slow activity; multifocal ictal discharges and subclinical ictal discharges. In cases with the secure diagnosis of RE, the documentation of an independent contralateral seizure onset may raise the suspicion of bilateral disease.

\section{Medical management}

Antiepileptic treatment, immunomodulatory treatment, steroids, immunoglobulins (IG), Plasmapheresis (PE) and immunosuppressive

${ }^{\star}$ Correspondence to: Krishnan Vijayan, Royal care super speciality hospital, Coimbatore, India, E-mail: kalyani_vijayan@rediffmail.com

Received: December 10, 2018; Accepted: December 19, 2018; Published: December 24, 2018 
therapy. Plasmapheresis useful in the treatment of status epilepticus, acute worsening of RE. Cyclo phosphamide. In 2009, a 20-year-old woman affected by RE had been suffering from EPC for 8 years and was successfully treated by Rituximab.

\section{Surgical management}

The principal surgical approach consists of disconnection of the affected hemisphere from the contralateral functioning hemisphere. Anatomical hemispherectomy. Hemidecortication. Hemi corticectomy. Transylvania keyhole functional hemispherectomy. Language performances remained preserve in the majority of cases due to the brain's plasticity. Thus, overall surgery preserves the cognitive abilities. Hemispherectomy should be carried out when the deterioration of cognitive functions continues, and there remains "something to save".

\section{Case report}

A 42-year-old women was in unusual status of health till 1999, when she had right focal onset convulsions with secondary generalisation. She was treated elsewhere for the same. MRI showed left frontal subcortical hyperintensity. Follow-up MRI showed new lesions misinterpreted as multiple sclerosis. She was started on anti-epileptic drug and was seizure free for a few months. She developed recurrent focal convulsions and was on multiple anti-epileptic drugs. EEG Showed sharp wave discharges over the left hemispheric montages. She presented to this hospital in January 2012 with worsening right hemiparesis and recurrent convulsions. MRI showed atrophy of left hemisphere with faintly enhancing white matter lesions (Figure 1A-D). She was awake, aphasic and abulic. She was on ryles tube feeds and sensorium was gradually worsening. She was stared on I.V. methyl prednisolone 1-gram OD for 5 days. Followed by oral methyl prednisolone. Oxcarbazepine $600 \mathrm{mg}$ TID and clobazam $10 \mathrm{mg}$ TID were continued. Sensorium improved. She started taking oral feeds and was saying a few words and phrases relevantly. She was discharged on an oral steroid taper with anti-epileptic Drugs. Fifteen days later she was readmitted with worsening sensorium. Review of the MRIs showed a progressive atrophy of the left perisylvian cortex (Figure 2A-C).

A FDG PET CT showed significant hypometabolism in left frontal cortex (Figure 3A-C). She was restarted on IV methyl prednisolone and a brain biopsy (right superior frontal gyrus) was done. Biopsy has confirmed Rasmussen's Encephalitis (Figure 4A-F). She was treated with Rituximab (500 mg/dose $\times 4$ doses 4 weeks apart) and was continued on Mycophenolate mofetil 500mg twice daily on which she still on. She has remained seizure free for the last 6 years with no further worsening of her motor symptoms. She is able to walk without support, speak a few words and remains seizure free till date.
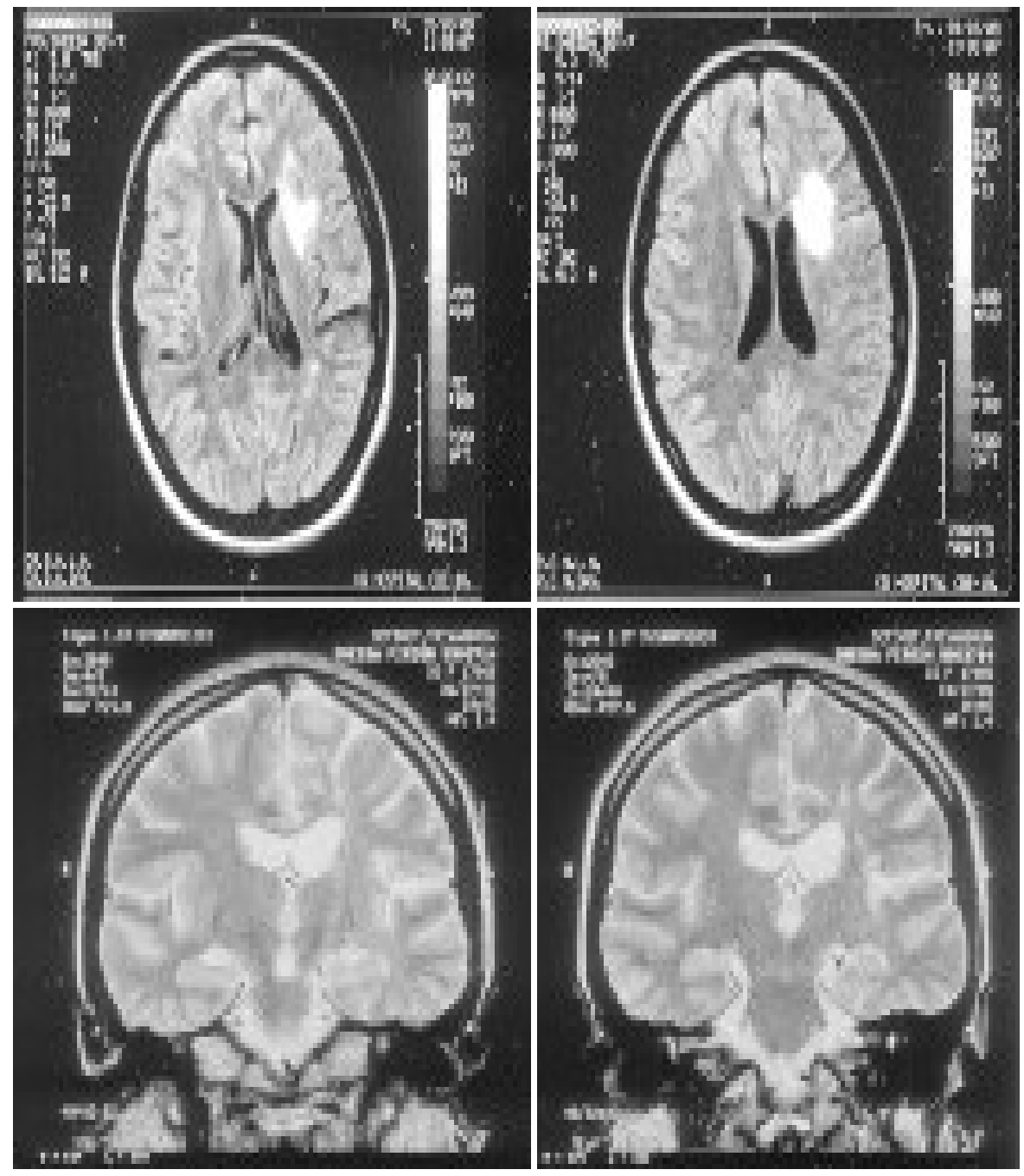

Figure 1. MRI showed left frontal subcortical hyperintensity (1999) 


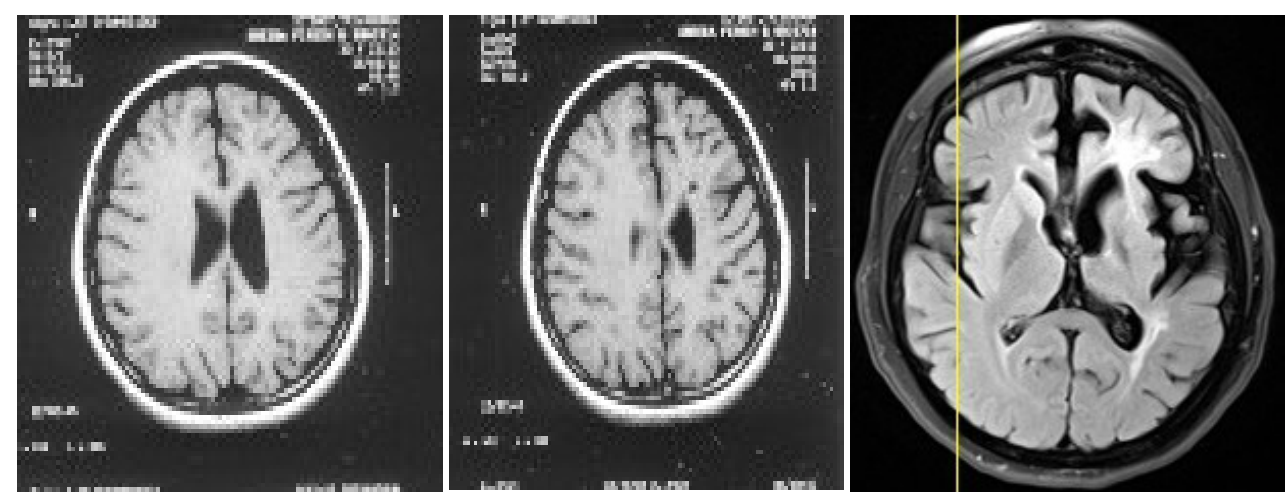

Figure 2. Review of serial MRIs showed progressive atrophy (2012)

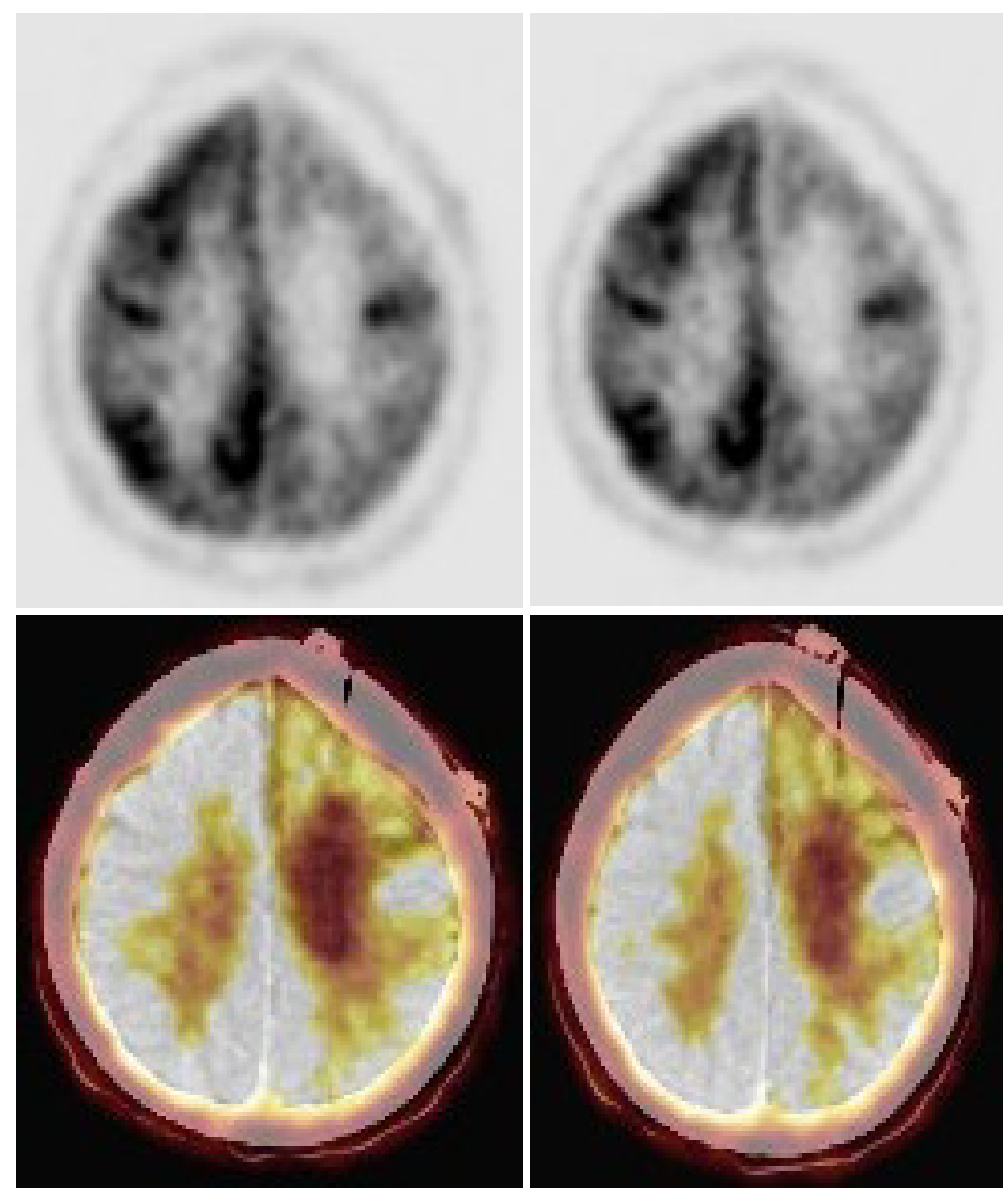

Figure 3. PET CT showed diffuse hypometabolism in the left 

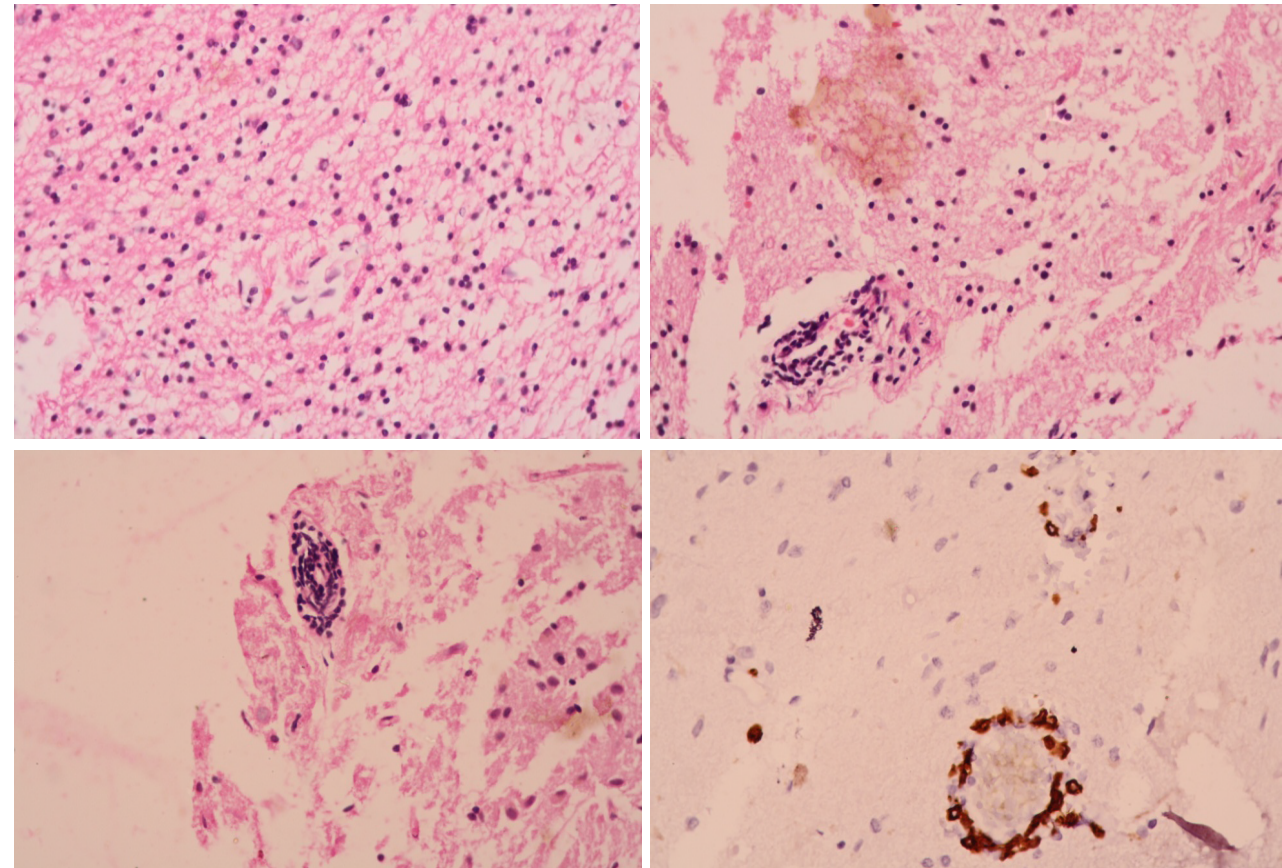

Figure 4. (A,B) Perivascular lymphocyte cuffing with spillage. (C) Perivascular lymphocyte cuffing. (D) Lymphocytes-cd3 staining. (E) Lymphocytes-cd8 staining perivascular interstitial. (F) Lymphocytes-cd3 staining

\section{Conclusion}

This case report is presented to highlight the rare presentation of Rasmussen encephalitis in adult and also successful immunotherapy with rituximab followed by maintenance mycophenolate mofetil.

\section{References}

1. Quesada CM, Urbach H, Elger CE, Bien CG (2009) Rasmussen encephalitis with ipsilateral brainstem involvement in an adult patient. J Neurol Neurosurg Psychiatry 78: 200-201. [Crossref]

2. Rasmussen T, Olszewski J, Lloydsmith D (1958) Focal seizures due to chronic localized encephalitis. Neurology 8: 435-445. [Crossref]
3. Veronica C, Ines DA, Jose C, Macarena G, Ignacio LJ, et al. (2016) Adult Rasmussen's Encephalitis. Clin Med Rev Case Rep 3:144.

4. Oguni H, Andermann F, Rasmussen TB (1992) The syndrome of chronic encephalitis and epilepsy. Adv Neurol 1992: 57: 419- 433. [Crossref]

5. Bien CG, Urbach H, Deckert M, Schramm J, Wiestler OD, et al. (2002) Diagnosis an staging of rasmussen encephalitis by serial MRI and histopathology. Neurology 58: 250-257. [Crossref]

6. Bien CG, Granata T, Antozzi C, Cross JH, Dulac O, et al. (2005) Pathogenesis, diagnosis and treatment of Rasmussen encephalitis. A European consensus statements. Brain 128: 454-471. [Crossref]

Copyright: (C2018 Vijayan K. This is an open-access article distributed under the terms of the Creative Commons Attribution License, which permits unrestricted use, distribution, and reproduction in any medium, provided the original author and source are credited. 